\title{
A Survey on the Prevalence of Zoonotic Helminthic Parasites in the Rats of Tabriz, Iran, Emphasizing the Importance of the Parasitic Fauna of Rats in the Public Health
}

\author{
Sahand Fattahi' ${ }^{1}$ Yagoob Garedaghi ${ }^{*}{ }^{*}$ \\ ${ }^{1}$ Postgraduate Student of Veterinary Medicine (D.V.M), Tabriz Branch, Islamic Azad University, Tabriz, Iran \\ ${ }^{2}$ Department of Parasitology, Tabriz Branch, Islamic Azad University, Tabriz, Iran
}

\begin{abstract}
Introduction: Parasitic diseases transmitted by rodents are more important among zoonotic diseases. On the one hand, rodents are the reservoirs of various parasites, including external parasites such as fleas, ticks, and mites, and some types of internal parasites such as cestodes and nematodes. On the other hand, they have a wide distribution and close relationship with humans which is important from the point of view of environmental health.

Methods: A total of 100 rats were caught from different parts of Tabriz in Iran. The trapped rodents were examined for species determination. Then, rats were anesthetized using chloroform and necropsied after recording the profile ensuring that this operation is carefully started from under the skin and all parts are examined to find possible fillers. Next, the abdominal and diaphragm muscles were evaluated for trichinosis, and finally, the entire gastrointestinal tract was separated and split along the length and in separate plates. They were placed together with the physiological serum and examined using a stereo microscope and a microscope. Eventually, smears were taken from the liver and spleen, and the esophagus was investigated for Gongylonema. Results: In this study, out of 100 collected rats, 68 (68\%) cases were infected with at least one worm, while $32(32 \%)$ of them were uninfected. The collected rodents were infected with Trichuris muris (9\%), Syphacia obvelata (2\%), Strongyloides ratti $(41 \%)$, Gongylonema (1\%), Physaloptera (8\%), and Nippostrongylus (3\%). Hymenolepis nana (18\%) and Hymenolepis diminuta $(62 \%)$, as well as Cysticercus fasciolaris (7\%) and Moniliformis moniliformis $(2 \%)$ were also detected in these collected rodents. Conclusion: The results of our study indicated the presence of Hymenolepis nana, Hymenolepis diminuta, Cysticercus fasciolaris, Moniliformis moniliformis, and Physaloptera as zoonotic worm parasites that have a history of infection in humans based on the findings of parasitological studies worldwide.

Keywords: Helminthic parasites, Zoonotic, Ratus, Tabriz, Public health
\end{abstract}

Received: March 5, 2021, Accepted: March 15, 2021, ePublished: April 1, 2021

\section{Introduction}

Parasitic diseases transmitted by rodents are more important among zoonotic diseases. This is because, on the one hand, rodents are the reservoirs of different parasites, including external parasites such as fleas, ticks, and mites, and contain types of internal parasites such as cestodes and nematodes. On the other hand, they have a wide distribution and close association with humans, and this is essential from the viewpoint of environmental health $(1,2)$.

Ratus are one of the most widely distributed pests in the world and are considered as important health pests. The biological characteristics of rodents, especially rats, have made them a reservoir of many pathogens, especially zoonotic parasites (3). Close human life in urban environments and poor health conditions with ratus colonies allows the transmission of common infections between humans and animals. Ratus mice have long been considered as health threats in human societies and zoonotic diseases that are common between humans and animals $(4,5)$. Among these diseases, we can mention pestilence, which has reduced the world's population at certain periods of history. Diseases from rats to humans can be transmitted through bites, contaminated claws, contact with urine and feces, as well as discharge from the mouth, nose, and hair. In addition, external rodent parasites such as ticks, lice, and fleas transmit a wide range of diseases to humans $(6,7)$. The above-mentioned cases highlight the importance of controlling, identifying, and combating diseases that jointly circulate between humans and animals, and rats can play the role of a reservoir in this regard. 
Some zoonotic worms (e.g., Hymenolepis nana) are common types, and other types (e.g., Hymenolepis diminuta, Acanthocephala, Capillaria hepatica), and in exceptional cases, Rectolaria worms can unusually infect humans (8).

Similar studies have been performed in different years and provinces of Iran to identify various rodent parasites. However, no comprehensive usable data are available on the parasitic fauna of this group of mammals, especially rats, in urban areas that are climatically highly different from other places. For this reason, this study focused on examining this part of the parasitology of Ratus mice in Tabriz, Iran.

\section{Materials and Methods}

This descriptive study started in the spring of 2021 and ended in the winter of 2021. In this study, it was attempted to perform sampling in all seasons of the year. A total of 100 rats were caught from different parts of Tabriz, Iran. Live traps were used to collect the rats so that the traps were planted at night and collected early in the morning. The trapped rodents were examined for species determination. It is extremely important to pay attention to different body sizes, especially the skull. In many cases, only a difference in size distinguishes species and races. Appearance traits (e.g., body hair color) are completely variable in different ages and conditions. The body size ratio is occasionally more valuable than the size itself. In this study, head and body size (from the tip of the nose to the base of the tail), tail (from the base of the tail to the end of the tail along the vertebrae without end hairs), hind legs (from the back of the heel to the tip of the longest toe without nails), and ears (from the top edge of the ear to the tip of the earlobe) were measured to determine rats.

Rats were anesthetized using chloroform and then necropsied after recording the profile ensuring that this operation is carefully initiated from under the skin and all parts were examined to observe possible fillers. Next, the abdominal and diaphragm muscles were investigated for trichinosis. Finally, the entire gastrointestinal tract was separated and split along the length and in separate plates. They were placed together with the physiological serum and evaluated using a stereo microscope and a microscope. Smears were also collected from the liver and spleen, and the esophagus was examined for Gongylonema.

The obtained parasites were kept in 10\% formaldehyde solution and $70 \%$ alcohol so that samples kept in $70 \%$ alcohol could be used for molecular tests for new species in necessary.

To detect worm samples, clarification and staining were performed, respectively. Lactophenol azocarmine and carmen acid staining were used in nematodes and cestodes, respectively. The final diagnosis was made using a Lucida camera microscope and a loop and according to morphometric and morphological features and prominent features based on Yamaguti and Skerjabine sources.

\section{Results}

In this study, out of 100 collected rats, 68 (68\%) animals were infected with at least one worm, but $32(32 \%)$ of them were uninfected.

The collected rodents were infected with Trichuris muris (9\%), Syphacia obvelata (2\%), Strongyloides ratti (41\%), Gongylonema (1\%), Physaloptera (8\%), and Nippostrongylus (3\%).

Hymenolepis nana (18\%) and Hymenolepis diminuta (62\%), as well as Cysticercus fasciolaris (7\%) and Moniliformis moniliformis (2\%) were detected in these collected rodents.

Based on the findings of this study, no worm contamination was observed in the lung, brain, and blood or the bladder tissue of the studied animals. Likewise, no Trichinella spiralis larvae were detected in any of the studied rodents when evaluated by pathological or other tissue digestion methods. The degree of the worm infestation of the caught rodents is presented in Table 1.

\section{Discussion}

Moridae rodents, especially rats, are able to adapt to diverse biological conditions and nutritional changes due to their biological characteristics. These characteristics, along with the high birth rate, have turned these creatures into the pests of urban and rural communities in most parts of the world. The omnivorous nature of these

Table 1. Worm Infestation of Ratus Mice Collected in Tabriz, Northwestern Iran

\begin{tabular}{lcccc}
\hline Nematoda & \multicolumn{3}{c}{ Cestode } & Acanthocephala \\
\hline Species & Percent & Species & Percent & Ppecies \\
\hline Trichuris muris & 9 & Hymenolepis nana & 18 & Moniliformis moniliformis \\
Syphacia obvelata & 2 & Hymenolepis diminuta & 62 & 7 \\
Strongyloides ratti & 41 & Cysticercus fasciolaris & & \\
Gongylonema sp. & 1 & & & \\
Physaloptera & 8 & & & \\
Nippostrongylus & 3 & & & \\
\hline
\end{tabular}

Note. H. nana: Hymenolepis nana; H. diminuta: Hymenolepis diminuta; M. moniliformis: Moniliformis moniliformis. 
rodents allows them to pose a threat to public health as a reservoir of some parasitic infections $(9,10)$.

Numerous and scattered studies on these small mammals in Iran over the past years have somehow shown the prevalence and distribution of zoonotic worms in some parts of the country $(11,12)$.

The present study was conducted in a part of Tabriz that has not so far undergone an investigation, and an information gap has been felt in this regard in this part.

According to the results of this study, indicating the presence of zoonotic parasitic worms, especially Hymenolepis diminuta and Moniliformis moniliformis in the gastrointestinal tract of these animals, the health importance of rodent population control in cities with similar conditions to the study area is highlighted more than ever.

Moniliformis moniliformis and Hymenolepis diminuta, which are in the list of the findings of this study, require the biological presence of common arthropods in the environment as intermediate hosts for transfer to their final host.

The presence of flour beetles (Tribolium castaneum) in food storage used to cook local snacks needs careful consideration. Moreover, most butterflies or even important cockroaches in public health are able to transmit Hymenolepis diminuta and Moniliformis to humans $(13,14)$.

Food hygiene and production monitoring in traditional food stores have always been a priority for health systems in most parts of the world $(15,16)$. Considering the obtained results and information about the transmission of zoonotic worms in rats to humans, the need for continuous monitoring regarding controlling this group of urban pests and implementing necessary health measures in the food production process, especially wheat flour products, rice, and to some extent oilseeds is getting further attention $(17,18)$.

The results of previous research revealed that the rate of infection with various types of domestic worm parasites, nematodes, cestodes, and trematodes varies in different regions of Iran (19).

\section{Conclusion}

Overall, the results indicated the presence of Hymenolepis nana, Hymenolepis diminuta, Cysticercus fasciolaris, Moniliformis moniliformis, and Physaloptera as zoonotic worm parasites that have a history of infection in humans based on the findings of parasitological studies worldwide.

\section{Acknowledgements}

We would like to express our deep gratitude to Islamic Azad University, Tabriz Branch, for offering valuable theoretical and practical assistance to the research team of the present study. Researchers sincerely thank all experts who provided the necessary cooperation for conducting this research.

\section{Authors' Contribution}

YG wrote and edited the manuscript, and SF collected and statistically analyzed data.

\section{Conflict of Interests}

The authors declare that they have no conflict of interest.

\section{Ethical Issues}

Ethical considerations were fully observed in this study.

\section{Funding}

The described results in this paper are part of an MSc thesis submitted by Sahand Fattahi. The study was financially supported by the office of the Vice-chancellor for the Research of Islamic Azad University, Tabriz Branch (Grant No. 162418312).

\section{References}

1. Seifollahi Z, Sarkari B, Motazedian MH, Asgari Q, Ranjbar MJ, Abdolahi Khabisi S. Protozoan parasites of rodents and their zoonotic significance in Boyer-Ahmad district, southwestern Iran. Vet Med Int. 2016;2016:3263868. doi:10.1155/2016/3263868

2. Kuhnen VV, Graipel ME, Pinto CJ. Differences in richness and composition of gastrointestinal parasites of small rodents (Cricetidae, Rodentia) in a continental and insular area of the Atlantic Forest in Santa Catarina state, Brazil. Braz J Biol. 2012;72(3):563-567. doi:10.1590/s151969842012000300019

3. Sadjjadi SM, Massoud J. Helminth parasites of wild rodents in Khuzestan province, southwest of Iran. J Vet Parasitol. 1999;13(1):55-56.

4. Harandi F. Survey parasit fauna of rodents in north of Isfahan. Tehran Univ Med Sci J. 1992;8:46-49. [Persian].

5. Hasanpuor H. Survey of worm infection in rodents whit emphasis on zoonotic species in Bandar Abbas. Tehran Univ Med Sci J. 2009;63:421-429. [Persian].

6. Meshke M. Survey of Ratus parasits whit emphasis on zoonotic species in Tehran. Tarbiat Modares Uni J. 2010;21:52-57. [Persian].

7. Stojcevic D, Mihaljevic Z, Marinculic A. Parasitological survey of rats in rural regions of Croatia. Veterinary MedicineCzech.2004; 49:70-74.

8. Forbes I. Rodent control in London's sewers. J R Soc Health. 1990;110(1):5-9. doi:10.1177/146642409011000102

9. Alborzi AR. Detection of Trichosomoides crassicauda, and Cysticercus fasciolaris Infection in Laboratory Rats in Ahvaz. Ahvaz, Iran: Proceedings of the 5th Congress of Iranian Veterinary Clinicians; 2007:87. [Persian].

10. Fish R, Danneman PJ, Brown M, Karas A. Anesthesia and Analgesia in Laboratory Animals. 2nd ed. American College of Laboratory Animal Medicine; 2008:139.

11. Garedaghi Y, Khaki AA. Prevalence of gastrointestinal and blood parasites of rodents in Tabriz, Iran, with emphasis on parasitic zoonoses. Crescent J Med Biol Sci. 2014; 1(1):9-12.

12. Yousefi A, Eslami A, Mobedi I, Rahbari S, Ronaghi H. Helminth infections of house mouse (Mus musulus) and wood mouse (Apodemus sylvaticus) from the suburban areas of Hamadan city, western Iran. Iran J Parasitol. 2014;9(4):511-518.

13. Soulsby EJ. Helminths, Arthropods and Protozoa of Domesticated Animals. 7th ed. London: Baillière Tindall; 19862:342.

14. Kamranrashani B, Kia E, Mobedi I, et al. Helminth parasites of 
Rhombomys opimus from Golestan province, northeast Iran. Iran J Parasitol. 2013;8(1):78-84.

15. Al Hindi Al, Abu-Haddaf E. Gastrointestinal parasites and ectoparasites biodiversity of Rattus rattus trapped from Khan Younis and Jabalia in Gaza strip, Palestine. J Egypt Soc Parasitol. 2013;43(1):259-268. doi:10.12816/0006382

16. Sarkari B, Hosseini G, Motazedian MH, Fararouei $M$, Moshfe A. Prevalence and risk factors of intestinal protozoan infections: a population-based study in rural areas of Boyer-Ahmad district, southwestern Iran. BMC Infect Dis. 2016;16(1):703. doi:10.1186/s12879-016-2047-4
17. Mansouri M, Sarkari B, Mowlavi GR. Helminth parasites of wild boars, Sus scrofa, in Bushehr province, southwestern Iran. Iran J Parasitol. 2016;11(3):377-382.

18. Chaisiri K, Chaeychomsri W, Siruntawineti J, Ribas A, Herbreteau V, Morand S. Diversity of gastrointestinal helminths among murid rodents from northern and northeastern Thailand. Southeast Asian J Trop Med Public Health. 2012;43(1):21-28

19. Nateghpour M, Motevalli-Haghi A, Akbarzadeh K, et al. Endoparasites of wild rodents in southeastern Iran. J Arthropod Borne Dis. 2015;9(1):1-6.

(C) 2021 The Author(s); This is an open-access article distributed under the terms of the Creative Commons Attribution License (http:// creativecommons.org/licenses/by/4.0), which permits unrestricted use, distribution, and reproduction in any medium, provided the original work is properly cited. 